J. Lake Sci. (湖泊科学) , 2015, 27(3):407-411

http://www. jlakes. org. E-mail : jlakes@niglas.ac.cn

(c) 2015 by Journal of Lake Sciences

\title{
硅藻群落结构的差异: 比较样本采集过程与空间梯度”
}

\author{
林金城 ${ }^{1}$, 蔡德所 ${ }^{1,2 * *}$, 姚文婷 ${ }^{1}$, 汤新武 $^{1}$, 钟云艳 ${ }^{2}$, 文宏展 ${ }^{3}$ \\ ( 1 :三峡大学水利与环境学院,宜昌 443002) \\ (2:广西大学土木建筑工程学院,南宁 530004) \\ (3:广西水环境监测中心, 南宁 530023 )
}

摘 要: 硅藻种群的分布与其所处的生境条件密切相关, 但在实际过程中的一些人为因素, 例如取样方法、样本的制备以 及藻种鉴定都可能会干扰到种群结构的分析结果. 因此很有必要探究这些人为因素是否会对真实的硅藻群落生态学研 究产生误导性的判断, 以免干扰硅藻生物水质评价的客观性. 选取采样方法及样本制备为代表的人为因素对硅藻群落生 态分析结果引人的误差大小进行评估. 结果显示, 子样本之间 (同一样品重复制作的玻片样本) 和样品之间 (同一样点重 复取样)群落结构差异大小分别是 $1.26 \%$ 和 $1.97 \%$,同一条河流的样点之间则为 $3.38 \%$,而所选定的跨河流研究区域的 样点间群落结构差异最大 $(42.03 \%)$. 生态学的排序结果和数理统计分析表明, 在硅藻群落结构分析中, 现场取样方法和 样本制备过程相对于不同生境条件所引起的变化,并不会带来较大的差异. 因此在河流附生硅藻的群落生态学研究中, 可基本不考虑取样方法等因子的干扰,而是侧重于环境因子对硅藻生态分布的影响.

关键词: 硅藻;群落结构;样本采集;空间梯度

\section{Variation in diatom community structures : Comparison between sampling procedures and spatial gradients}

\author{
LIN Jincheng ${ }^{1}$, CAI Desuo ${ }^{1,2}$, YAO Wenting ${ }^{1}$, TANG Xinwu ${ }^{1}$, ZHONG Yunyan ${ }^{2}$ \& WEN Hongzhan ${ }^{3}$ \\ (1: College of Hydraulic and Environmental Engineering, China Three Gorges University, Yichang 443002, P. R. China) \\ (2: College of Civil Engineering and Architecture, Guangxi University, Nanning 530004, P. R. China) \\ (3: Water Environment Monitoring Center of Guangxi Province, Nanning 530023, P. R. China)
}

\begin{abstract}
The distribution of diatom is closely related to the habitat characteristics. But some anthropogenic factors, such as sampling method, sample preparation and species identification may affect the result of analyzing community structure. It is necessary to ensure no misleading judgments that would be generated by these factors in the diatom community ecology study because such misleading judgments may affect the evaluation of water quality. This paper was to quantify the amount of variability in community structure of benthic diatom, which related to sampling method and sample preparation. The results showed that the variability in community structure was minimal among sub-samples (repeated microscope slides of the same sample) and samples (repeated sampling at the same site), $1.26 \%$ and $1.97 \%$, respectively. Differences in community structure were $3.38 \%$ at two sites located in the same river. Amount of dissimilarity in community structure across the streams was up to $42.03 \%$. Multivariate analysis suggested that field sampling and sample preparation did not contribute to a large amount of variation in our analysis of diatom community structure that related to habitat conditions across sites. Therefore, in the study of diatoms community in river areas, emphasis should be put on the effects of environments rather than the factors about sampling methods.
\end{abstract}

Keywords: Diatoms; community structure; sampling; spatial gradients

在河流水质监测中,利用水生生物对周围环境因子变化的响应,分析其生理功能、种类丰度、种群密度、

* 国家自然科学基金项目(40971280, 30870345)资助. 2014-07-17 收稿;2014-10-12 收修改稿. 林金城( 1988～), 男,硕士研究生;E-mail:jchlin@ foxmail.com.

** 通信作者;E-mail:caidesuo@vip. 163. com. 
群落结构等特征, 能够较全面地反映水体在一段时间内的污染状况, 在一定程度上补充了传统水质监测的 不足 ${ }^{[1-3]}$. 近年来, 生物监测在河流健康评价体系中发挥着重要作用 ${ }^{[4]}$, 其灵敏度高、综合性强等特点 ${ }^{[5]}$, 已 被许多国家纳人本地区河流健康诊断与治理计划中,并且也得到成熟运用.

许多学者对底栖硅藻的研究表明, 硅藻与流速、水深、 $\mathrm{pH}$ 、营养元素、电导率等理化因子具有一定的相关 性 ${ }^{[6-10]}$, 同时也会受到土地利用、地形地貌、季节变迁、植被和气候变化的影响 ${ }^{[1-1-14]}$. 基于底栖硅藻的水质评 价中, 仍存在一些不确定因子会干扰实验结果, 例如空间变化、时间变化、取样方法、样本的制备、样本的分 析、研究人员因素等, 最终会影响分析评价的客观准确性 ${ }^{[15-18]}$. 本文将从取样方法及样本制备的角度出发, 分别采集同一条河流的不同样点和跨河流的硅藻样品, 评估一些人为因素引起群落结构的差异大小, 也可 为广西桂北区域的河流硅藻生物水质评价提供一定的参考意义.

\section{1 材料与方法}

\section{1 样点概况及设置}

为进行此次验证性工作,将分别从漓江、贺江、刀江、龙江和贝江中采集硅藻样品. 这些江都受到不同程 度的人为活动干扰, 包括农林业活动、城镇化、水利建筑设施, 其中贺江、龙江、习江上游局部地区采矿业较 密集,发生过重金属污染事件.

位于漓江的阳朔设为 $\mathrm{A}\left(24^{\circ} 47^{\prime} 01^{\prime \prime} \mathrm{N}, 110^{\circ} 29^{\prime} 42^{\prime \prime} \mathrm{E}\right)$, 贺江的大宁样点设为 $\mathrm{B}\left(24^{\circ} 33^{\prime} 47^{\prime \prime} \mathrm{N}, 111^{\circ} 54^{\prime} 23^{\prime \prime} \mathrm{E}\right)$, ᄀ江的下考乡样点设为 $\mathrm{C}\left(24^{\circ} 40^{\prime} 12^{\prime \prime} \mathrm{N}, 107^{\circ} 44^{\prime} 20^{\prime \prime} \mathrm{E}\right)$, 龙江的宜州样点设为 $\mathrm{D}\left(24^{\circ} 30^{\prime} 26^{\prime \prime} \mathrm{N}, 108^{\circ} 35^{\prime} 52^{\prime \prime} \mathrm{E}\right)$, 贝江的等塘和沟滩样点分别设为 $\mathrm{E}\left(25^{\circ} 13^{\prime} 06^{\prime \prime} \mathrm{N}, 109^{\circ} 10^{\prime} 04^{\prime \prime} \mathrm{E}\right)$ 和 $\mathrm{F}\left(25^{\circ} 08^{\prime} 46^{\prime \prime} \mathrm{N}, 109^{\circ} 13^{\prime} 23^{\prime \prime} \mathrm{E}\right)$ (图 1). 其 中 $\mathrm{A}$ 点为旅游景区, 河流底质主要以我卵石、砾石、细砂等沉积物, 水流条件较好且水体透明度高, 植被覆盖 率较好, 受到一定人为活动干扰; $\mathrm{B}$ 点与 $\mathrm{D}$ 点处在城镇河段上游, 河流底质主要是大石、碎石, 水体透明度较 高, 水流条件较好,但各自水量都受到上游水利枢纽的影响,附近植被覆盖率一般; $\mathrm{C} 、 \mathrm{E} 、 \mathrm{~F}$ 点两岸基本是经 济林区, 河岸稳定, 河流底质为鹅卵石、砾石、细砂等沉积物, 水流条件较好, 流量不大, 水体清澈, 属浅水型 河流, 周围植被覆盖率高.

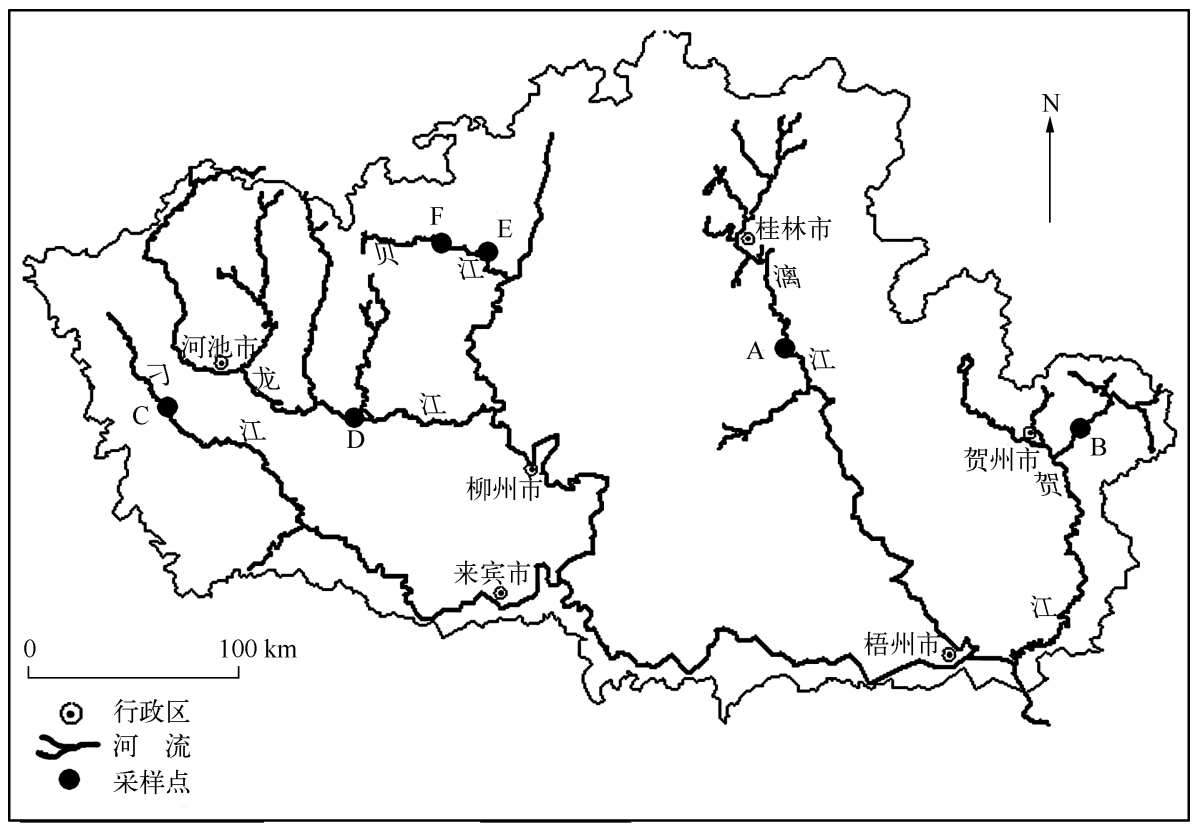

图 1 采样点位置

Fig. 1 Location of sampling sites in the rivers 


\section{2 样品采集及处理}

于 2014 年 3 月对上述 5 条河流进行硅藻采样. 参考欧州技术标准 EN $13946^{[19]}$, 样点选择在地势开阔 且无树荫遮挡处, 选取能抵抗一定水流冲击的石头, 每个样品至少选取 5 块石头, 采集范围控制在大约 $5 \mathrm{~m}^{2}$ 内,并用干净的牙刷刷洗石头的向阳面,再装人样品瓶中,加人甲醛 $(3 \% \sim 4 \%)$ 固定保存. 最后带回实验室, 经过氧化消解后, 制成合格的永久玻片, 每个样品重复相同步骤制成 5 个玻片, 并分别编号为 $A_{1-5} 、 B_{1-5} 、 \mathrm{C}_{1-5} 、$ $\mathrm{D}_{1-5}$, 共有 20 个玻片. 另外, 在 $\mathrm{E}$ 和 $\mathrm{F}$ 样点中, 分别采集两组样品, 每组沿着垂直河流水流方向, 水深依次由 浅到深,采集 5 个样品,每个样品采集方法和上述一样,但采集范围控制在大约 $2 \mathrm{~m}^{2}$ 内. 每个样品分别制作 成一个永久玻片,并分别编号为 $\mathrm{E}_{1-5} 、 \mathrm{~F}_{1-5}$,一共有 10 个玻片.

\section{3 硅藻鉴定计数及分析}

参考欧洲技术标准 EN $14407^{[20]}$, 在光学显微镜下采用 1000 倍油镜鉴定, 视野内所有硅藻及破损面积 不超过 $1 / 4$ 的样品都要进行鉴定和计数, 每个玻片鉴定的总数大于 400 个. 硅藻种类的鉴定主要根据 Krammer 和 Lange-Bertalot 的鉴定体系 ${ }^{[21]}$.

采用 DCA(CANOCO4.5) 分析硅藻群落结构与样品采集及处理过程中的变量关系, 排序分析过程中剔 除每个样点里相对丰度小于 $1 \%$ 的藻种, 并对其进行平方根转换. 现将所有样本分成两组进行 DCA 分析 (第 1 组 $A_{1-5} 、 B_{1-5} 、 C_{1-5} 、 D_{1-5}$ 和第 2 组 $E_{1-5} 、 F_{1-5}$ ), 再对 DCA 中每个物种的得分进行 ANOVA(SPSS 18.0) 分析, 将所得方差分量与 DCA 中各轴的解释量相乘, 相加后的和便是本组样本在不同尺度下群落差异的实际大 小. 另外为比较河流间、同一条河流不同样点间、同一样点不同样品间、子样本间的差异大小, 以第 1 组差异 大小作为参考量, 将第 2 组 $\left(\mathrm{E}_{1-5} 、 \mathrm{~F}_{1-5}\right)$ 中群落结构的分析值乘以比例 $(\text { 第 } 2 \text { 组特征值/第 } 1 \text { 组特征值 })^{[22]}$.

\section{2 结果}

对第 1 组样本 (分别从 4 条不同河流中采集的样品,并将每个样品制成 5 个玻片) 进行 DCA 排序分析的 结果可知, 总特征值为 1.679 , DCA 轴 1 解释了群落结构变化的 $35.7 \%$, 轴 2 解释了群落结构变化的 7.6\% (图 2). 将 DCA 轴 1 得分进行 ANOVA 分析, 发现河流间和子样本间的硅藻群落结构方差分量分别占 $99.02 \%$ 和 $0.98 \%$, 其中 $F(3,16)=538.787, P<0.001$, 很显然河流间的群落结构差异远远大于子样本间的 差异; 将 DCA 轴 2 得分进行 ANOVA 分析, 发现河流间与子样本间硅藻群落结构方差分量分别占 $87.97 \%$ 和 $12.03 \%$, 其中 $F(3,16)=38.969, P<0.001$, 也说明河流间的群落结构差异大于子样本间的差异. 因此, 河 流间的硅藻群落结构实际差异大小为 $99.02 \% \times 35.7 \%+87.97 \% \times 7.6 \%=42.03 \%$, 子样本间实际差异大 小为 $0.98 \% \times 35.7 \%+12.03 \% \times 7.6 \%=1.26 \%$.

对第 2 组样本 (从第 5 条河流中两个样点采集的 10 个样品,并将每个样品制成 1 个玻片)进行 DCA 排 序分析的结果可知, 总特征值为 0.228 , DCA 轴 1 解释了群落结构变化的 $39.3 \%$, 轴 2 解释了群落结构变化 的 $12.2 \%$ (图 3). 将 DCA 轴 1 得分进行 ANOVA 分析,显示样点间与样品间的硅藻群落结构方差分量分别 占 $63.25 \%$ 和 $36.75 \%$, 其中 $F(1,8)=13.741, P=0.006$, 样点间的群落结构差异大于样品间的差异; 将

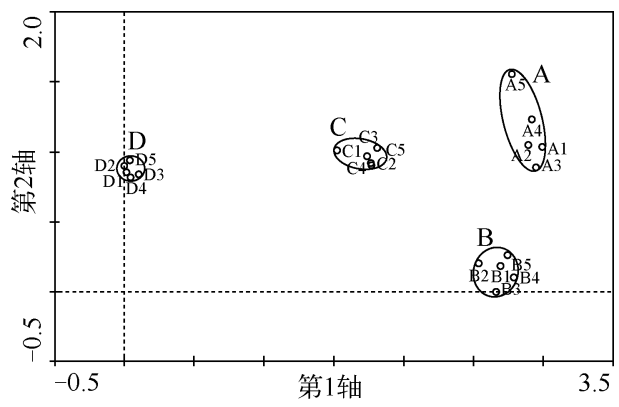

图 2 每条河流的 5 个玻片样本的 DCA 排序结果

Fig. 2 DCA scores from the 5 slides for each stream

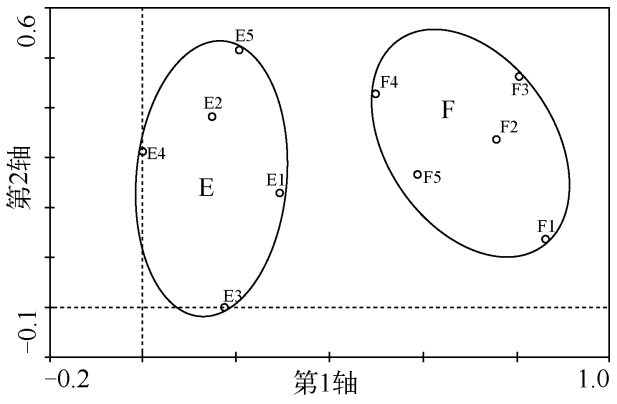

图 3 贝江中每个样点 5 个样本的 DCA 排序结果

Fig. 3 DCA scores from the 5 samples collected at 2 sites in the Bei River 
DCA 轴 2 得分进行 ANOVA 分析, 显示样点间与样品间硅藻群落结构方差分量分别占 $1.91 \%$ 和 $97.71 \%$, 其 中 $F(1,8)=0.169, P=0.691$, 无显著差异. 因此, 样点间的硅藻群落结构实际差异大小为 $63.25 \% \times$ $39.3 \% \times 0.228 / 1.679=3.38 \%$, 样品间实际差异大小为 $36.75 \% \times 39.3 \% \times 0.228 / 1.679=1.97 \%$.

综上所述, 采样和样品处理分析过程中引起的群落差异比较为:跨河流间差异 $(42.03 \%)>$ 同一条河流 不同样点间差异 $(3.38 \%)>$ 相同样点的不同样品间差异 $(1.97 \%)>$ 同一样品的子样本间差异 $(1.26 \%)$.

\section{3 讨论与结论}

就样品采集和处理过程对硅藻群落结构对比, 发现除了在河流间、样点间会出现群落结构差异, 在样品 间及子样本间的群落结构也不是完全一致的, 只是差异很小.

这 4 条河流间的硅藻群落结构差异性达到了 $42.03 \%$, 河流中的生物群落, 除了受到水体理化因子的影 响外,与其所处生态分区也有一定的关系 ${ }^{[23]}$. A 点所在的漓江属于旅游景区, 周围的生境条件良好; B 点所 在的贺江受采砂、农田及城镇化等干扰; C 点所在的习江受上游地区采矿及沿岸农田影响, 且河流的径流量 较小; $\mathrm{D}$ 点所在的龙江与ᄀ江相邻, 受到城镇化、采矿活动及梯级电站开发影响. 另外河流之间相隔较远, 由 于空间效应 ${ }^{[24]}$, 导致这些河流间群落结构差异性较大. 在第 5 条河流贝江中, E、 $\mathrm{F}$ 两个样点间的差异性只 有 $3.38 \%$, 两样点的群落结构相似性较高, 这跟其流域特征有一定的关系, 贝江流域面积相对较小, 年径流 量也不大, 作为柳州水源地, 其水质条件整体优良 ${ }^{[25]}$, 两个样点间的河段人口密度小, 两岸翠山环抱, 植被覆 盖率好, 也没有水利建筑设施等人为干扰, 基本处于天然状态. 除了生境条件比较接近外, 可能还跟这两点 相隔的距离比较近有关, Hillebrand 等发现硅藻种群的相似性随着距离的增加而减小 ${ }^{[26]}$, 两个样点群落差异 性的可能因素是物种自身传播 ${ }^{[27-28]}$ 以及与其他生物的互相作用 ${ }^{[29]}$, 同一河流相隔较远的样点群落结构差异 性会比较明显. 而在同一个样点的 5 个样品间以及同一样品制成的 5 个玻片间的群落结构差异大小分别为 $1.26 \%$ 和 $1.97 \%$, 引起差异性的原因可能是由于人员对藻种的鉴定带有一定主观性 ${ }^{[30]}$, 另外样品采集 ${ }^{[31]}$ 和 制作玻片过程中也可能存在一些偶然因素. 在整个数据采集过程中, 尽管会引起一些误差, 但是其差异性大 小都不足 $2 \%$,基本可以忽略这些因素带来的影响.

来自不同基质的硅藻种群也会有差异性, Kitner 等 ${ }^{[32]}$ 发现附石、附植、附泥沙中硅藻种群多样性有较大 不同, 但 Winter 等 ${ }^{[33]}$ 和 Cejudo-Figueiras 等 ${ }^{[34]}$ 则认为种群结构差异受基质影响不明显. 为了避免因基质不 同对结果产生干扰, 所有样点统一选取石头上的硅藻. 另外 Mykrä 等 ${ }^{[31]}$ 报道了在芬兰北部河流中的硅藻群 落与季节变迁有关, $O^{\prime}$ Driscoll 等 ${ }^{[35]}$ 在北爱尔兰 Burrishoole 流域却未发现本地区硅藻群落的季节性变化, 这 可能是流域频发洪水的水文特征导致的. 此次实验数据只反映了春季的情况, 属于平水期, 在其他季节, 不 同尺度下的种群差异性是否会增大或者减小, 在文中未进行验证, 但样点间差异大于样品间差异的结论, 与 Kelly ${ }^{[36]}$ 对比硅藻群落结构在年际间的变化差异结果是一致的.

本文评价了样品采集和处理过程中可能带来的误差大小. 跨河流及不同样点间的群落差异较明显, 这 主要是因为水体中的理化条件不同, 而样品采集和样本制备中出现的偶然因素对群落结构的干扰远小于理 化因子、生态分区、空间距离等因素的影响.

\section{4 参考文献}

[1 ] 房英春, 刘广纯, 田 春等. 浅析河流水体污染的生物监测及指标生物. 水土保持研究, 2005, 12(2): 151-153.

[ 2 ] 赵彦伟, 杨志峰. 河流健康: 概念、评价方法与方向. 地理科学, 2005, 25(1) : 119-124.

[ 3 ] 王 静, 池玉林, 陈正西. 利用底栖动物进行水质监测与评价的研究进展. 安徽农业科学, 2012, 40(22): 1140111405 .

[ 4 ] 汪 星, 刘录三, 李 黎. 生物监测在跨界河流中的应用进展. 中国环境监测, 2014, 30(2): 170-177.

[ 5 ] 阴 琨, 吕怡兵, 滕恩江. 美国水环境生物监测体系及对我国生物监测的建议. 环境监测管理与技术, 2012,24 (5) : 8-12.

[ 6 ] van Dam H, Mertens A, Sinkeldam JA. Coded checklist and ecological indicator values of freshwater diatoms from the Netherlands. Netherlands Journal of Aquatic Ecology, 1994, 28: 117-133.

[ 7 ] 吴乃成, 唐 涛, 周淑婵等. 香溪河小水电的梯级开发对浮游藻类的影响. 应用生态学报, 2007, 18 (5): 1091-1096. 
[ 8 ] 蔡德所, 黎佛林, 文宏展. 广西金鸡滩库区水深梯度下的硅藻生态特征研究. 三峡大学学报: 自然科学版, 2014, 36 $(2): 1-5$

[ 9 ] Ponader KC, Charles DF, Belton TJ. Diatom-based TP and TN inference models and indices for monitor-ing nutrient enrichment of New Jersey streams. Ecological Indicators, 2007, 7 (1) : 79-93.

[10］邓培雁, 雷远达, 刘 威等. 桂江流域附生硅藻群落特征及影响因素. 生态学报, 2012, 32(7): 2196-2203.

[11] Leland HV. Distribution of phytobenthos in the Yakima River basin, Washington, in relation to geology, land use and other environmental factors. Canadian Journal of Fisheries and Aquatic Sciences, 1995, 52 (5) : 1108-1129.

[12] Hlúbiková D, Novais MH, Dohet A et al. Effect of riparian vegetation on diatom assemblages in headwater streams under different land uses. Science of the Total Environment, 2014, 475 : 234-247.

[13] Drira Z, Elloumi J, Guermazi W et al. Seasonal changes on planktonic diatom communities along an inshore-offshore gradient in the Gulf of Gabes (Tunisia). Acta Ecologica Sinica, 2014, 34 (1) : 34-43.

[14] 刘 彩, 刘道辰, 陈诗越等. 附着硅藻的研究进展. 聊城大学学报:自然科学版, 2013, (3) : 47-51.

[15] Kelly M, Bennion H, Burgess A et al. Uncertainty in ecological status assessments of lakes and rivers using diatoms. Hydrobiologia, 2009, 633 (1) : 5-15

[16] Barbour MT, Gerritsen J, Snyder BD et al. Rapid bioassessment protocols for use in streams and wadeable rivers: Periphyton, benthic macroinvertebrates, and fish. Washington, DC: Environment Protection Agency, 1999.

[17] Clarke RT. Estimating confidence of European WFD ecological status class and WISER Bioassessment Uncertainty Guidance Software (WISERBUGS). Hydrobiologia, 2013, 704(1) : 39-56.

[18 ] Bennett C, Owen R, Birk S et al. Bringing European river quality into line: an exercise to intercalibrate macroinvertebrate classification methods. Hydrobiologia, 2011, 667 (1) : 31-48.

[19] EN 13946: Water quality-Guidance standard for the routine sampling and pre-treatment of benthic diatoms from rivers, 2003.

[20] EN 14407: Water quality-Guidance standard for the identification, enumeration and interpretation of benthic diatom samples from running waters, 2004.

[21] Krammer K, Lange-Bertalot H. Bacillariophyceae. Berlin: Spektrum Akademischer Verlag GmbH Heidelberg, 2000.

[22] Lavoie I, Somers KM, Paterson AM et al. Assessing scales of variability in benthic diatom community structure. Journal of Applied Phycology, 2005, 17(6) : 509-513

[23] Soininen J. Determinants of benthic diatom community structurein boreal streams: the role of environmental and spatial factors at different scales. International Review of Hydrobiology, 2004, 89(2) : 139-150.

[24] Heino J, Bini LM, Karjalainen SM et al. Geographical patterns of micro-organismal community structure: are diatoms ubiquitously distributed across boreal streams? Oikos, 2010, 119(1) : 129-137.

[25] 唐 金菳, 蔡德所, 黎佛林等. 贝江硅藻生态群落特征及水质评价. 三峡大学学报: 自然科学版, 2013, 35(6): 1-5.

[26] Hillebrand H, Watermann F, Karez R et al. Differences in species richness patterns between unicellular and multicellular organisms. Oecologia, 2001, 126(1) : 114-124.

[27] Smucker NJ, Vis ML. Spatial factors contribute to benthic diatom structure in streams across spatial scales: Considerations for biomonitoring. Ecological Indicators, 2011, 11(5) : 1191-1203.

[28 ] Černá K. Small-scale spatial variation of benthic algal assemblages in a peat bog. Limnologica-Ecology and Management of Inland Waters, 2010, 40(4) : 315-321.

[29] Borcard D, Legendre P. Environmental control and spatial structure in ecological communities: an example using oribatid mites (Acari, Oribatei). Environmental and Ecological Statistics, 1994, 1(1) : 37-61.

[30 ] Prygiel J, Carpentier P, Almeida S et al. Determination of the biological diatom index (IBD NF T 90-354) : results of an intercomparison exercise. Journal of Applied Phycology, 2002, 14(1): 27-39.

[31] Mykrä H, Saarinen T, Tolkkinen M et al. Spatial and temporal variability of diatom and macroinvertebrate communities: How representative are ecological classifications within a river system? Ecological Indicators, 2012, 18 : 208-217.

[32] Kitner M, Poulícková A. Littoral diatoms as indicators for the eutroPhication of shallow lakes. Hydrobiologia, 2003, 506/ 507/508/509(1/2/3) : 519-524.

[33] Winter JG, Duthie HC. Stream epilithic, epipelic and epiphytic diatoms: habitat fidelity and use in biomonitoring. Aquatic Ecology, 2000, 34(4) : 345-353.

[34] Cejudo-Figueiras C, álvarez-Blanco I, Bécares E et al. Epiphytic diatoms and water quality in shallow lakes: the neutral substrate hypothesis revisited. Marine and Freshwater Research, 2010, 61(12) : 1457.

[35 ] O'Driscoll C, Eyto E, Rodgers M et al. Spatial and seasonal variation of peatland-fed riverine macroinver-tebrate and benthic diatom assemblages and implications for assessment: a case study from Ireland. Hydrobiologia, 2014, 728 (1): 67-87.

[36] Kelly MG. Role of benthic diatoms in the implementation of the urban wastewater treatment directive in the River Wear, north-east England. Journal of Applied Phycology, 2002, 14(1) : 9-18. 